\title{
The Endogenous Mode of Beautiful Rural Construction: A Case of China
}

\author{
Liaoji Zheng ${ }^{*}$, Gang Li², Yiyan Guo ${ }^{3}$, Huanyu Wang ${ }^{4}$ \\ ${ }^{1}$ School of Management, Eastern Liaoning University, Dandong, China \\ ${ }^{2}$ School of Design, Eastern Liaoning University, Dandong, China \\ ${ }^{3}$ School of Agriculture, Eastern Liaoning University, Dandong, China \\ ${ }^{4}$ School of Management, Eastern Liaoning University, Dandong, China \\ Email: ^zhengliaoji@vip.sina.com
}

How to cite this paper: Zheng, L. J., Li, G. Guo, Y. Y., \& Wang, H. Y. (2020). The Endogenous Mode of Beautiful Rural Construction: A Case of China. Journal of $\mathrm{Hu}$ man Resource and Sustainability Studies, 8 , 349-370.

https://doi.org/10.4236/jhrss.2020.84020

Received: October 11, 2020

Accepted: November 30, 2020

Published: December 3, 2020

Copyright $\odot 2020$ by author(s) and Scientific Research Publishing Inc. This work is licensed under the Creative Commons Attribution International License (CC BY 4.0).

http://creativecommons.org/licenses/by/4.0/ Open Access

\begin{abstract}
[Objective] An endogenous mode (EM) for beautiful rural construction (BRC) considers the transformation of rural endogenous energy, and the rational choice of a Rural Revitalization Strategy (RRS). The driving force creating the endogenous mode comes from an actor network process, which is composed of new technology, new industry, and new business forms. The EM is also a process by which the combination of innovations by multiple actors replaces traditional simple exogenous innovation. [Methods] Focusing on Dalishu village (DLS) in China as a case study, this research analyzed the characteristics of endogenous energy transformation in an actor-network node into an endogenous mode of BRC, using a social network analysis method. This paper proposes the theoretical framework of an EM for BRC, which integrates all stakeholders, and promotes the transformation of the BRC mode from materialized productivity to the post-productivity of shared experiential services. [Result] EM is closely related to actor-network construction. The more centrally located the actor node is, the greater the transformation energy is, the higher the transformation efficiency is. The stronger the central potential of the entire network is, the stronger the sustainability of the endogenous mode is. [Conclusion] When considering the five strategic objectives of supply side structural reform, it is important to adjust the strong and weak relationships among different actors, strengthen the interaction among actors across network nodes, and improve the high-quality efficiency of EM. Cultivating actors with high transformation efficiency, strengthening the relationship between network participants, and planning and building a shared space can promote the role and change the status of actors, improving the efficiency of EM.
\end{abstract}

\section{Keywords}

Beautiful Rural Construction (BRC), Endogenous Mode (EM), Social 
Network Analysis (SNA), Dalishu Village (DLS)

\section{Introduction}

China's rural areas have undergone significant adjustments under the influence of contemporary globalization and national intervention. Driven by urbanization, informatization, Beautiful Rural Construction (BRC), and other factors, rural space has continued to diversify in differentiated ways. Gradual national-level intervention in rural development has focused on restructuring the agricultural sector and rural China, to improve participation in globalization and to prepare for the industrialization and modernization of rural areas. As the creative executor of national policy and the coordinator of local action, local governments directly impact the agricultural, social and spatial restructuring of rural areas (Chen et al., 2019).

With the acceleration of urbanization and a series of top-down reforms, China's rural socio-economic and spatial structure has experienced tremendous adjustments. Rural development has gradually shifted from the exogenous development of economic growth to a more "people-oriented" endogenous development mode. In this "self-directed" development process, rural areas can achieve the expected form of development and realize the redistribution of the comprehensive value of the rural area. From the development of BRC to the proposal of "Rural Revitalization" strategy, China's management of rural areas has gradually risen from local practice to national strategic level, and BRC has become a necessary way to narrow the gap between urban and rural areas.

$\mathrm{BRC}$ is also affected by external factors, with self-organization and government intervention being alternative methods to guide rural transformation. $\mathrm{Na}$ tional policy is a fundamental driving force of BRC, but self-organization plays an even more important role (Zang et al., 2020). In addition to strategic level implementation, the endogenous mode (EM) also depends on the rural area's internal factors. The networking mode, which depends on the active participation of the stakeholders in the rural area, also becomes an important basis for EM development. This network mode fully considers the interdependence of the rural economy and society, and emphasizes the sustainable utilization of different social forces and resources. The core idea of endogenous development is to see development as a bottom-up process, which acknowledges the key role of regional actors and civil society groups (Tödtling, 2020).

With the development of global industrialization and urbanization, the theory of endogenous development involves an increasing number of actors and influences, gradually extending to all fields of rural development. The EM involves the innovation of space production, which refers to the production of subjects and objects in space and the production of space itself, and the gradual networking of industrial integration, social governance, regional cultural construction, and ecological environment protection. These are the driving forces behind 
the endogenous development of BRC. The EM construct suggests that full attention be given to the enthusiasm of community participation in governance, with particular attention paid to the leading role of rural sages in activating the development potential in rural areas. We should pay attention to the development of the local economy, and consider the revitalization of rural social culture.

As a "self-directed" process, the new EM should facilitate resource integration with agricultural production, social governance, landscape construction, ecological environment, cultural construction, and other aspects in a sustainable direction. This would achieve the expected goal of building environment-friendly, beautiful, and livable environments, and realize the continuous optimization of rural life, production, and ecological space (Zheng, 2010). The Ministry of Agriculture of the People's Republic of China has released ten modes for constructing beautiful villages in China. These modes include the industrial development mode, ecological protection mode, suburban intensive mode, social comprehensive management mode, cultural inheritance mode, fishery development mode, grassland pasture mode, environmental remediation mode, leisure tourism mode, and efficient agriculture mode. All of these provide references for a BRC.

As time passes, EM also faces the pressure of demands for space production and industrial integration, social governance, ecological construction, and other changes. This is due to the acceleration of China's industrialization and urbanization, which has led to significant rural population outflow. This, in turn, has created a series of "rural hollowing out" problems, such as idle rural cultivated land, a weak rural cooperative collective economy, single forms of agricultural production, and a lack of rural civilization. The scattered distribution of agricultural production and the transformation of traditional agricultural production modes are important factors affecting agricultural transformation. In the context of RRS implementation, traditional agriculture needs to pay more attention to diversifying the livelihoods of rural small-scale farmers to achieve agricultural transformation (Ge et al., 2020).

The presence of a number of different problems also shows that the formation of EM is a nonlinear and gradual process, including the participation of different stakeholders. In addition to agricultural integration, the composition and structure of the rural governance system also determine the direction of beautiful rural development. In particular, the change in mode determines the direction of rural development (Zang et al., 2020). In contrast with other rural areas, agriculture in the Pearl River Delta region of China has mainly improved the living environment and natural ecological environment, by developing aquaculture and forestry. Therefore, the rural areas in the Pearl River Delta have become a mode of world development (Tang, 2019).

However, regardless of the form of EM, rural development cannot rely on a single mode alone. The ecological economic conditions are the important foundation for constructing and transforming the EM. The limitations of local production, life, and ecology need to be fully considered, changing the choices local people make from a single form of element to diversified elements. This enriches 
the many values of rural spatial production. The characteristics of the social, cultural, and ecological environment also promote a community-based collaborative mode led by the government to gradually transform the mechanism into a combination of external and endogenous forces.

The force that most drives adjustments in the rural spatial structure differs in different stages. This includes the interaction and co-existence of different elements in the government, enterprises, residential and tourism market. The driving force of EM mainly comes from the integration and innovation between primary industry (e.g., planting), secondary industry (e.g., food processing), and tertiary industry (e.g., service industry). EM driven by rural tourism promotes land consolidation and is a direct way to adjust rural spatial structure (Gao \& Cheng, 2020). Rural tourism has been recognized as a key approach for rural development and poverty reduction, and high-quality and efficient rural tourism is also a comprehensive and sustainable mode of rural revitalization (Gao \& $\mathrm{Wu}$, 2017).

The theory of economic transformation refers to the transition from one economic operating state to another. There are four key elements in this transformation: the target mode, the initial conditions, the process, and the ultimate conditions. Forming a new EM is a network process that involves the agricultural mode, local culture adjustments, ecological pattern reconstruction, and the development process of each actor that integrates into the network. A prominent feature in transformation activities is that the rural endogenous mode has changed from materialized agricultural production to providing both material production and experiential services. EM mainly depends on the active integration of knowledge, technology, information, data, and other actors. This helps realize the mutual transformation of explicit knowledge and tacit knowledge in rural areas, and helps develop innovative knowledge with regional characteristics.

With the wide application of the mobile Internet, cloud computing, big data, artificial intelligence, the Internet of Things (IoT), blockchain, and other new generation information technologies in agriculture and rural economic and social development, the EM can contribute to the high-quality development of the rural economy, the digitization of social governance, and the globalization of cultural and tourism integration. By cultivating and strengthening new driving forces and new modes, rural areas have transformed from the traditional mode to an innovative new mode. This has advanced relevant elements to continuously integrate into the new mode of the network, leading the rural mode through a transformation from low- to high-level. In this new mode, the role of node actors in the network changes greatly, and the interaction between actors and other network nodes forms new modes.

The new development mode fully reflects a "five in one" development concept, including ecology, politics, economy, culture, and society. The choice and formation of EM also relate to the rational choice of a diversified rural lifestyle. This rational choice based on the transformation of old and new modes pro- 
motes the symbiosis of government leadership, multi-stakeholder participation, multiple forms of oversight, and multiple actors. In this network of livelihood choices, integrating ecology, society, economy, culture, history, aesthetics, and other functions, the transformation of innovative knowledge with multifunctional agriculture as the core is particularly important for constructing beautiful villages in China (Zheng \& Liu, 2018; Zheng et al., 2019).

\section{Theoretical Framework}

\subsection{Construction of the Endogenous Mode}

EM is conducted through social network construction, which links changes in form and the self-renewal of knowledge transformation with different types of social capital, and promotes interactions between network node actors. The actor-network process formed by EM plays a positive role in improving the attitude of local residents in supporting a diversity of livelihoods, enriching community participation, and expanding professional organizations. Moreover, this improves the image perception ability of rural landscape space reconstruction. Providing comprehensive social services for rural innovation activities helps enhance the rural nature of the landscape space, and promotes the establishment of coupling linkages among agricultural production factors, rural life elements, and ecological elements (Zheng et al., 2019). Establishing a coupling mechanism between economy, society, ecology, and culture is an important rational choice process to promote the value diversification of EM. This mechanism can also eliminate low-level situations where there is growth, but no development caused by "Involution", which refers to the phenomenon that a social or cultural model will stagnate or fail to transform into another advanced mode after reaching a certain form at a certain stage of development.

From an economic perspective, establishing new modes is a process of changing old and new modes, and replacing underdeveloped productive forces with advanced productive forces. Although there is strong uncertainty, there is ultimately a change from a matter orientation of productivism to a more service orientation of post-productivism, with the goal of achieving a modern multifunctional agricultural system. This reflects the accumulation and growth of a new agriculture, new technology, new business forms, and new mode. It also represents the network's ability to promote the transformation of a rural economy from being factor driven to innovation driven, making modern agriculture a pillar industry in rural green sustainable development.

From a macro perspective, the driving force of the new mode mainly comes from the coupling linkage of technological innovation, factor upgrades, deepening reform and opening up, and consumer demand. From a development perspective, the transformation of new and old modes open multiple paths, reflecting changes from government-led to government-guided, from economic field to social field, from specific demonstration to comprehensive promotion, from factor-driven to innovation-driven, and from gradual innovation to disruptive 
innovation. From the perspective of China's agricultural development, land system reforms facilitate the release of farmers' energy and rural social elements, facilitating the transformation of the rural development mode, and realizing the symbiosis of rural life, production, and ecology. Scientific and technological innovation, industrial integration, industrial space integration, and cultural tourism integration interact to produce the power of network construction, and further integrate the coordinating relationships between external driving forces and the behavior and power of endogenous forces. This improves the quality and efficiency of BRC.

The energy of EM comes from rational choices, which are the results of individual purposeful behavior. These behaviors include individual or collective actors acting or interacting in the most favorable way to meet goals, given the resources and environment. Economic research assumes that rational choices reflect many types of choices, with economic rationality occupying a dominant position. With an increasing number of factors involved in EM, decision-making has become increasingly complex, and other forms of rationality have become important choices in development. The decision-making behavior of EM reflects gradual changes from economic rationality to rational choices related to life, social dynamics, ecology, and culture. It also aligns decision-making behavior with the needs of urban-rural integration.

In every rational choice, some development factors restrict the direction of value diversification, resulting in diversified outcomes. At the same time, people's rational choices are affected by preference, belief, judgment, will and character, and values. This leads to different judgments and choices. From the perspective of social governance, rural areas have experienced many modes of governance: centralized and unified, rural autonomy and multiple forms. This has continuously improved the comprehensive governance level of rural areas. The comprehensive effect of political, economic, clan, cultural, and ecological elements has led to a rural social governance structure with complex and diversified characteristics.

EM adapts to the evolution of these elements and facilitates a singular focus on economic function towards a more spatial production mode, integrating culture, society, ecology and life. This new spatial production mode improves the diversity of rural life, facilitates the construction of livable space, encourages shared experiential activities, and improves high-quality construction. The transformation of factors determined by rational choices clarifies BRC goals and results in rationally diversified spatial production. This enriches the internal network structure of BRC.

In the process of EM networking, actors transform the identity of different rural elements, optimize the industrial structure, and improve the rural governance structure by "exchanging the old for the new." EM expands the development context of BRC and evolves into a "de-intervention" sustainable development path. BRC relies on the social ecological system, which is composed of the 
social subsystem, ecological subsystems, and their interaction. Together, these manifest different structures, functions, and complex characteristics from the individual systems. The coupling system formed by the mutual feedback of society and ecology is complex, nonlinear, uncertain, and multi-layered. These characteristics drive rural endogenous development (Cumming \& Stephan, 2018).

EM transformation involves upgrades from the current state to another state, eliminating stagnation. Otherwise, it cannot transform into a new advanced "involution" mode, coordinating the kinetic energy transformation of actors and creation of space to achieve high-quality BRC development. The essence of EM transformation is a change in the development concept: from the productivism of material production, to the post-productivism of service provision. Rural areas are no longer engaged in pure material production, but rather, material production and shared experiential services. The essence of shared experience includes material and service provisions on the supply side, and the perceptions of experience on the demand side. The supply side relies on both the traditional industrial chain to meet the demand side experience, and on the integration of different elements to form a complex network. This integrates the supply side and the demand side, effectively transmitting multiple values.

It is important to consider the rational and social effects of different forms of business, to advance the rational development of the rural economy, and avoid the influence of rational and social policies on the development of new types of business. As an increasing number of elements begin to mutually connect, the traditional agricultural chain presents complex and diverse network forms. This creates more abundant and diversified values. The nodes in rural network become the actors with a mode transformation effect. This strengthens the equal exchange relationship between urban and rural elements, creating a new important force for a new industrial and new development mode, and facilitating the diversification of rural residents' livelihood. The innovation energy of the BRC lies in promoting the transformation of EM in industrial, ecological, production, life, societal, and cultural elements. EM is an important indicator to measure the implementation level of RRS, which is an important topic for this study.

Guided by the five major RRS goals of "innovation, coordination, green, open, and sharing," this study constructed an EM theoretical framework (Figure 1) involving actors in the dimensions of industrial development, cultural construction, ecological protection, social governance, and civilization construction. This promotes linkages among different actors and achieves the purpose of promoting the BRC. According to ANT, networking includes the process of redefining, ranking, and endowing the interests, roles, functions, and status of each actor in the network; however, it also reflects the sharing behavior of interest exchanges among actors (Rodger, Moore, \& Newsome, 2009). An actor's status, role, and exchange interests on the network nodes are related to the structure, function, and goal of the network. The network is also affected by the interaction between 


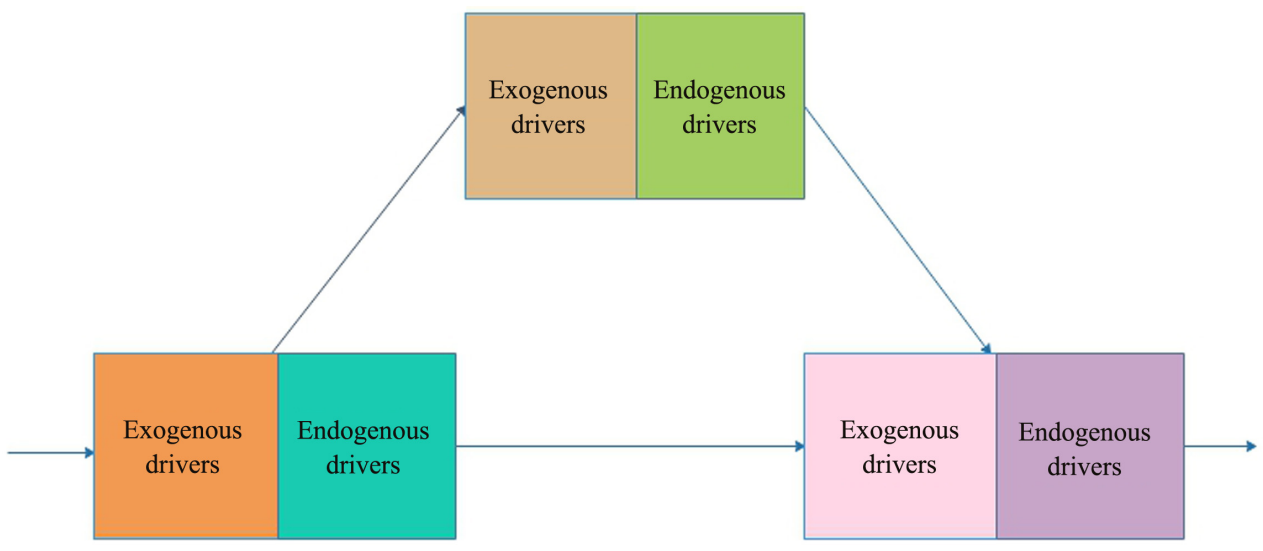

Figure 1. The driving process of actor-network in beautiful rural construction.

other actors. These actors have reached a certain "consensus" through networking, promoting a "common" rational choice. This "consensus" rational choice coordinates the different elements and agricultural production resources involved in rural development. This state also helps solve the problems of rural development homogenization, industrial chain shortening, weak industrial integration ability, and single business model. The transformation and upgrading of rural tourism promote BRC and rural spatial justice, and has become the preferred "consensus" of mode transformation.

The new EM does not mean that rural development requires giving up the urban connection with the external region. Instead, it should strengthen the connection with the city and obtain the external energy to match the endogenous development in an appropriate way. The new endogenous mechanism effectively transforms tangible or intangible resource elements into the land, technology, environment, system, and other elements. These elements can be transformed into products that can generate a lasting income stream, and construct the transformation mode of resources and environment assets of the BRC.

Rural tourism creates a primary network of life, production, ecology, society, and culture. This network process is inseparable from the overall social space, which is full of cultural differences, conflicts, fractures, and diversity. The rural experience space is constructed by an ecological culture, which includes the ecological environment, rural landscape, and folk customs. The new rural space generated by the interaction of different actors provides inexhaustible power and sources of innovation to develop human civilization, social progress, and economic prosperity. This should ultimately improve the comprehensive competitiveness of the rural area.

\subsection{Actor-Network of Beautiful Rural Construction}

A BRC actor-network is a mechanism by which EM achieves bottom-up revitalization and development, by relying on endogenous forces. The actor-network has the representational significance of material space, economic benefits, social benefits, and other dimensions. It is an important basis for analyzing whether 
rural areas conform to the endogenous development. According to actor network theory (ANT), any heterogeneous factors that can change the state of elements are actors, and they may be tangible or intangible. This heterogeneity of actors eliminates any opposition between human and non-human, and eliminates the binary opposition between nature and society. It also allows actors to maintain a complete symmetry of status and role in the process of network construction (Ai \& Miao, 2010). In an actor-network, all boundaries are broken and there is no difference between the upper and lower levels, and between the natural and social. Each actor is free to connect in an equal relationship.

The interaction of actors in the network constitutes the energy of interaction, and becomes an important foundation for network stability. The BRC actor-network considers the social relations among actors, and the complex relationships among different types of actors, such as land use, rivers, folk customs, animals, and plants (Law, 1992; Steen, 2010). The EM mechanism lies mainly in changes of the key actors' intentions, the adoption of rural governance mechanisms, and the change in the actor's role in the process of actor-network translation. Actors can constantly adjust the power relationship with others to realize the sustainable development of space production and modern multifunctional agriculture (Wang \& Xiao, 2020). The EM can mitigate knowledge gaps at the institutional level, and constructing an informal network can create more innovation opportunities for rural food production enterprises, and continuously improve the participation of different actors (McKitterick et al., 2016).

In the BRC process, actors and networks maintain the relationship of time and space. By connecting through agriculture, social governance, cultural connection, and other forms of spatial connection, the actors and network construct a new pattern of rural multifunctional agricultural development ( $\mathrm{Li}, \mathrm{Fu}, \& \mathrm{Liu}$, 2014). The different social factors of the BRC include the interactive relationship established by social organizations, the ability to learn, the degree of participation, and the governance mode. These fully reflect the adaptability and transformation ability of actors in the network. Social organizations influence residents' adaptation and behavioral changes through their connectivity to the social network. Learning ability is the adaptive ability that people acquire by interacting with the ecological environment and surrounding people, and helps them judge whether behavior should be changed. Community participation is the adaptive behavior that occurs when social governance changes local residents' cognition (Barnes et al., 2020).

Introducing ANT into EM broadens the research focus from an external to internal perspective, from a global scale to local scale, from a material form to cultural form, from linear time to physical space, and from container space to interactive network space. Application of the theory facilitates the transformation of rural mode towards sustainable direction (Allen, 2011). In the process of constructing a BRC actor-network, different elements of production, life, and ecology are integrated into the network and transformed into actors. As such, all kinds of actors become an important force to facilitate the formation of new 
formats and new modes in rural areas, and expand rural industrial structure, spatial functions, and social functions (Zheng, 2018). Through the transformation of knowledge, spatial functions, and value, actors with exogenous and endogenous motivation continuously amplify effects across the network, and cause the network process to move in the direction that supports the actors playing the greatest role. The internal micro-circulation of each actor is consistent with the large cycle formed by network construction, which supports overall network stability. BRC actor-network construction occurs when different actors establish interaction links, and when actors exerting their power. Actors involved in the network can obtain energy from other actors in the network and exert external energy on other actors, making the actors more closely related (Figure 2).

BRC actor-network construction is formed by rational choices, and reflects the directional behavior of actors in the network. Rational choice theory is a

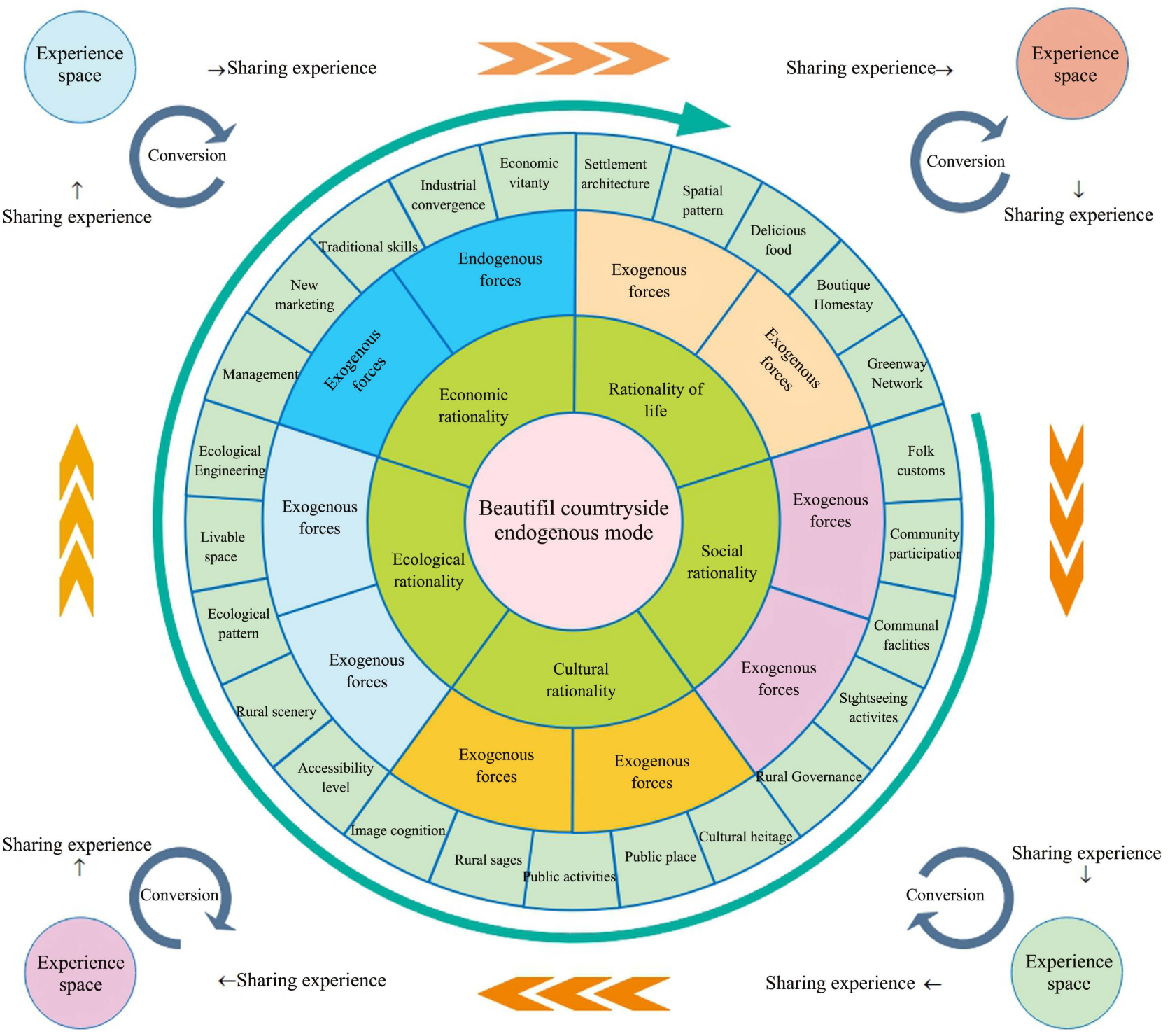

Figure 2. The theoretical framework of endogenous mode in beautiful rural construction. 
leading theoretical paradigm in many social science fields, and is a framework for understanding and often formally simulating social and economic behaviors. Owing to different cognitive, biological, and environmental constraints, local rationality is the tendency of human beings to reason using local and contextual information, which may lead to global inconsistencies in situational and domain behavior (Vlaev, 2018). The theory of rational choice proposes that, in the process of social choice, actors use norms and environmental conditions or resources to make the best choice among different behaviors or objects. This leads to differences in the functions and values of rural economic and social ecosystems. External and internal factors in the traditional agricultural mode affect the choices farmers make with respect to livelihood diversity, making it difficult to meet the survival needs of a local society.

Adjustments in the agricultural structure is generally a first choice in transforming the rural mode. Farmers adjust the structure of traditional farming, reduce the consumption of natural resources, and adopt diversified modes to maintain local farmer livelihoods (Yin, Yang, \& Chen, 2020). In many rural areas, pursuing high-yield agricultural products has become a basic feature of agriculture, however, this is difficult to achieve with the limitations of the traditional mode (Gautam \& Andersen, 2016). Natural capital, social capital, and other forms of livelihood capital owned by farmers play an important role in their adaptive livelihood behavior. In reality, not every actor can control all the resources needed to meet their own interests, and many natural and social resources are generally controlled by others (Kuang et al., 2019).

Scientific rationality is a scientific research standard integrating philosophy, sociology, and decision-making science. Rational choices that are consistent with local practice reflect public "consensus" and form a more adaptive EM. The premise underlying rational choice is to maximize the utility of individual interests, however, each actor may control some resources needed by others. The control dynamics change as urbanization unfolds. The imbalance in China's economic growth has led to a gap between urban and rural economic development. Under the influence of urban and rural economic and social changes, rural spatial structures have experienced many internal and external transformations. This transformation is also an important reason for rural residents to move to urban areas to engage in high-income jobs.

In rural areas dominated by agriculture, the main way to change the development mode is to break actor dependence on the traditional path. This involves carrying out integration and innovation from different aspects of industry, society, culture, ecology, and human settlement. It also means attracting a more urban population and rural floating population, encouraging people to return to their hometown to start their own businesses; facilitate the integration of rural primary industry, secondary industry, and tertiary industry; and form new business formats and new development mode, to narrow the gaps between urban and rural areas (Chen \& Wang 2019). The new EM formed by urban-rural integration is reshaping a new value relationship between urban and rural areas, at- 
tracting more capital and makers to rural areas, and facilitating the two-way flow of people, logistics, and information (Chen et al., 2020).

The BRC actor-network structure consists of exchange relationships, trust relationships, a trust system, normative structure, and collective "consensus". This structure influences the social exchange behavior of different rural resources, and changes the status and role of each actor. In a simple social exchange relationship, there are four types of actors: actors with "control", "interest", "strength", and "value". In the networking process, each type of actor obtains a position in the network through the exchange of initial interests and resource value and power. This usually manifests as social and economic status. Actors change their status and role during networking, transforming the network's structure and function. The transformation of actors at each level has a certain purpose or direction, with the goal of realizing the preferred values and utility they pursue. This transform acquired external and internal forces into a certain network status. This kind of overall behavior, composed of the rational choice behavior of individual actors, also represents the pursuit of goals in the network translation process and becomes the starting point of network development.

This represents the logical starting point of this paper. By virtue of their status and influence in the network, different actors can motivate others to recognize their own goals and requirements. This can achieve a certain level of consensus and recognition of the normative rights of other actors. By voluntarily exchanging resources that are not affected by external factors, actors in the network can obtain more benefits from the exchange, and the specific interests of the involved party remain undamaged. In addition, the best balance of social and economic exchange can be achieved through the transformation process involved in networking.

Social normative behavior, followed by networking, respects the macro domain rights of other actors. Each actor realizes a transformation from micro to macro, establishing a relatively stable social network relationship. According to rational choice theory, many economic activities are rooted in all aspects of social life. In other words, economic activities cannot be separated from the constraints or support of social norms. As the main body of rural development, stakeholders are an important force in RRS. Through a series of ecological economic activities, stakeholders constantly optimize the structure of rural social space production and gain the multifunctional value of multifunctional agriculture and space production. Several micro objectives generally support each macro goal; in other words, rational choices usually involve several sub-items to coordinate. Together, these form the dominant direction of rational choice. The macro goal of RRS is to achieve rural revitalization, by integrating five micro-objectives: industry, life, ecology, governance, and society.

\section{Research Methods}

\subsection{Research Area}

Dalishu village (DLS) is in the eastern mountainous area of Liaoning Province. It is characterized by a temperate monsoon climate, with four distinct seasons. 
There is abundant rainfall in summer, dense green vegetation, rich rivers and streams, an excellent ecological environment, and a beautiful landscape. The village has a total population of 4800 , with a total area of $4800 \mathrm{hm}^{2}$, a cultivated area of $495 \mathrm{hm}^{2}$, and a forest coverage of $3596 \mathrm{hm}^{2}$.

DLS has experienced five modes over the past 40 years: traditional agriculture marketization, the centralization of the settlement space, the serialization of leisure agriculture, multifunctional agriculture networking and shared experience modernization, and spatial production patterns guided by tourism (Figure 3). Different mechanisms have dominated each stage, with innovative governance methods, marketing methods, and the integration of technology. This has realized the transformation of the development mode. The five development stages of DLS have been a process of continuous mode transformation of industrial integration, space production, social governance, and cultural advancement. Based on BRC, the landscape is becoming more livable, promoting the sharing of experiences between local people and outsiders. In addition to industrial integration, a series of activities such as annual sports events, farmer harvest festivals, and food festivals are held to enrich cultural and tourism activities.

The state has awarded the DLS the distinction of being an $A A A A$ scenic spot, national agricultural tourism demonstration area, and Chinese agricultural park. The area has developed into a famous beautiful village in China, which is also an outcome of adjustments in internal and external forces. Because of its innovative characteristics, DLS has become a BRC model in the Eastern mountainous area of Liaoning Province. The development of the rural settlement form and agricultural

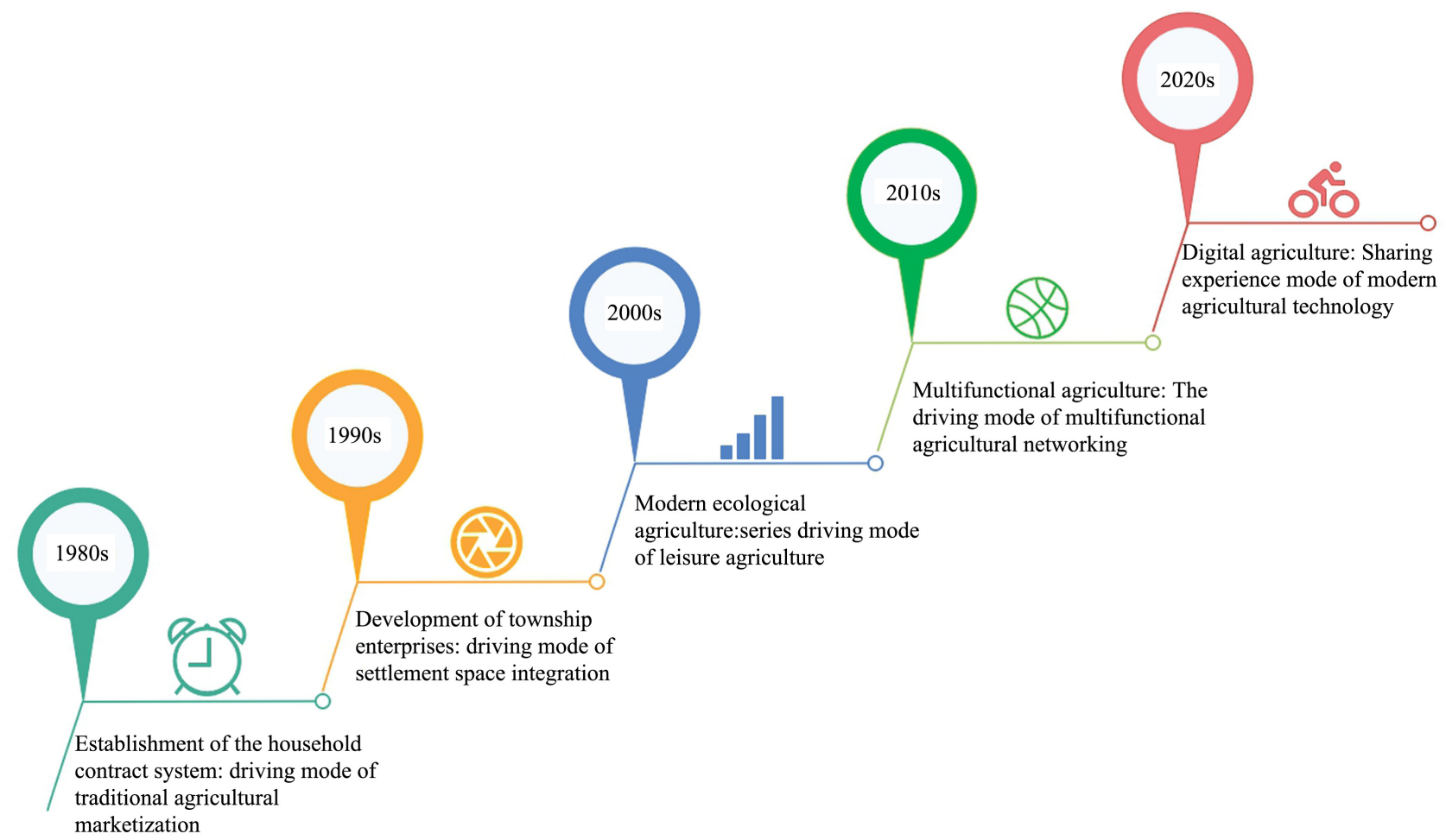

Figure 3. The mode transformation stage of Dalishu village. 
creative landscape has become an important symbol of the integration of production, life, and ecology. This improves the level of multifunctional agricultural production and social governance, and continuously enhances external contacts and demonstration effects. The process of EM shows that leadership achieved the endogenous development of the village using actor-level networking, rather than using a top-bottom approach. Moreover, farmer cooperatives (such as Schisandra chinensis professional cooperatives) help promote the participation and development of local communities and a relatively fair distribution of interests.

Rural tourism has been an active driving force, advancing land consolidation and transforming innovative knowledge into an important basis for modal changes. The local people organically integrate explicit, tacit and self-transcendent knowledge about the rural landscape space. This enables them to construct different types of ecological experience space for innovative knowledge transformation, build a relatively stable and secure ecological landscape, and improve the ecological service level and the value of the beautiful countryside (Zheng, 2017).

\subsection{Data Acquisition}

This study used field investigations and interviews, which combined media and face-to-face interviews, and network media and data on agricultural production, community participation, characteristic landscape, creative agriculture, and the ecological environment. These data explain the characteristics of landscape ecology, settlement architecture, social governance, other knowledge elements, agricultural production, and folk customs of DLS. Based on the five dimensions of BRC, 25 actors were selected to establish a $25 \times 25$ correlation matrix to verify the theoretical framework of EM.

\subsection{Social Network Analysis}

Social network analysis (SNA) is a mature analysis method in rural sociology. It decomposes the relationship structure between social actors and reveals interdependencies between different individual behaviors and social outcomes. This is also referred to as the structure of social relations. When studying network relationships, it helps to combine the relationships between individuals, the micro-network, and the macro-structure of the large-scale social system. This quantitative analysis method has been widely used in sociology. In this context of this study, different business entities have been embedded into rural society and have established complex economic and social relations with the local community. This has become an important basis for rural innovation and development (Richter, 2019). Social innovation is closely related to "new endogenous" growth. The new EM requires a good balance between local initiative and resources, as well as appropriate investments in capital, expertise, and innovation sources. In the start-up stage, social innovation depends highly on rural community members, civil society organizations, and local public departments. 
In the implementation stage, the main body of social innovation consists of non-governmental organizations (Jungsberg et al., 2020).

From an SNA perspective, the interactions between people in a social environment can be expressed as a mode of social structure based on relationships. This allows for a quantitative analysis that is the starting point of SNA. It is both an analytical tool and a mode of relational thinking. This study proposed and validated a theoretical framework to identify key actors that significantly impact EM. The degree of centrality is used to measure the centrality of actors in the network, and network centralization is used to measure the concentration of network actors. A greater degree of node centrality indicates that the greater the influence of actors on other actors in the network is, the more important their status is, the greater their capabilities are.

The measurement formula of the standardized degree of centrality is as follows:

$$
C_{D}^{\prime}\left(N_{i}\right)=\frac{C_{D}\left(N_{i}\right)}{g-1}
$$

Equation (1), $C_{D}^{\prime}\left(N_{i}\right)$ represents the degree of centrality of node $i$. The variable $\mathrm{g}$ is the number of nodes, or, the number of actors in the BRC.

This research applied the software program UCINET 6 to calculate the standardized in-degree centrality and out-degree (Huang \& Yu, 2010). The in degree measures the effect of external forces on the actor, that is, the dynamic effect of other actors on the implementation of the actor, while the out degree is the action of the actor on the others, that is, the dynamic effect on other actors. The energy transformation efficiency of each actor is as follows:

$$
Q=\frac{N_{r m} \text { OutDeg }}{N_{r m} \text { OutDeg }+N_{r m} \text { InDeg }}
$$

Equation (2), Q is the energy transformation efficiency of every actor (\%); $N_{r m}$ OutDeg is the standard point out degree, representing the actor's conversion energy; and the $N_{r m}$ InDeg represents the external energy obtained by the actor. The $Q$ value expresses the proportion of energy transformation of actors, indicating the ability of the actor to transform the energy of other actors.

\section{Results and Conclusion}

\subsection{Results}

1) EM reflects the comprehensive network behavior, including the interaction of different internal actors and the integration of external forces. The form of the EM depends on the centrality of actors at each node and the centralization of the network. Network cohesion is stronger when the network is more concentrated. This also increases the efficiency of energy transmission between actors, and the strength of the energy transformation. Community participation, image recognition, management, rural sages, and rural governance were found to be the most powerful actors in this research's case study. Image recognition, public activities, 


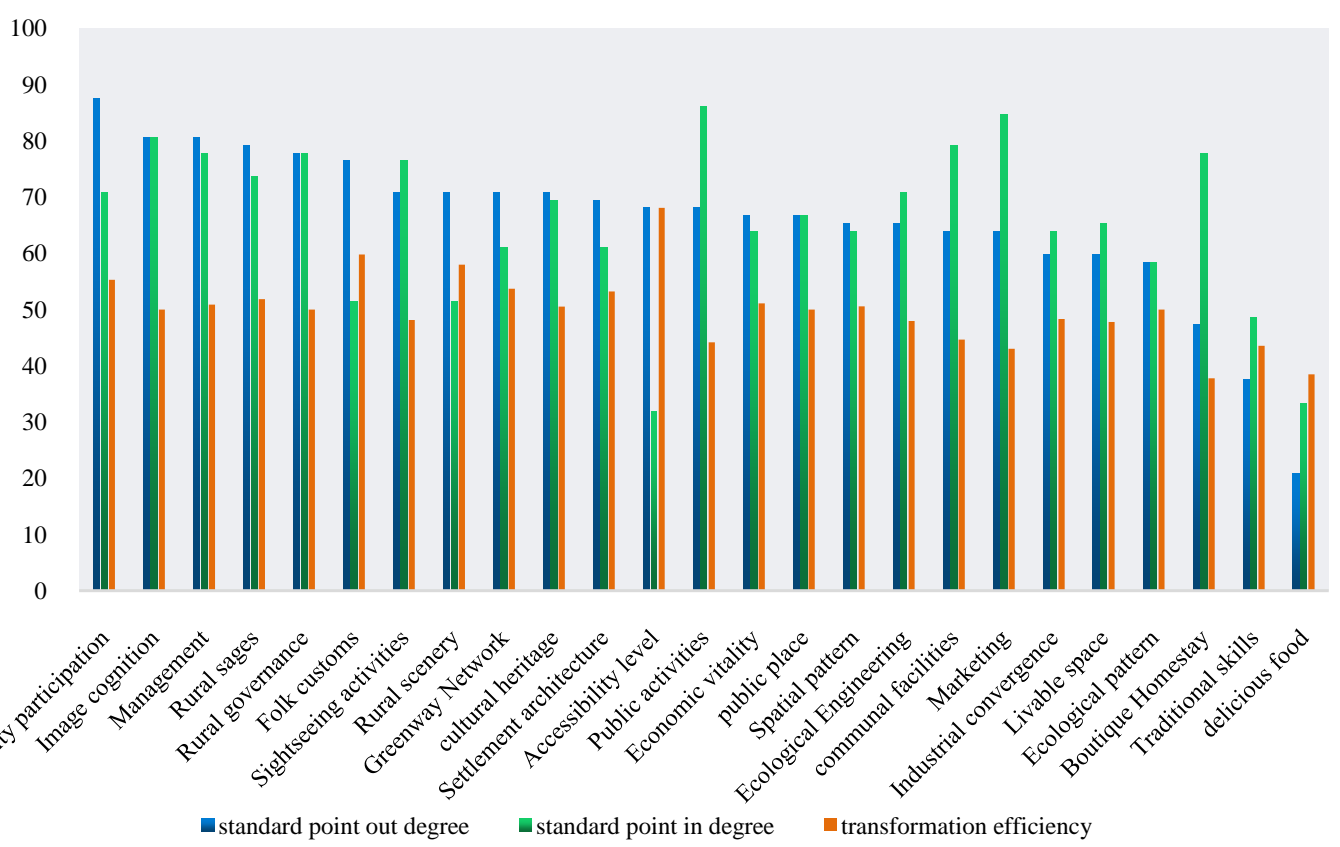

Figure 4. In degree, out degree and transformation efficiency of actors.

and marketing were the most active actors in the DLS rural actor-network (Figure 4). Community participation, image recognition, and management were the three actors with a standardized point out degree $\geq 80$. Community participation, image recognition, management, rural sages, rural governance, ecological engineering, and boutique homestay were the main actors, with a standardized point in degree $\geq 70$. The main actors with a standardized point out degree $\geq 70$ included rural sages, rural governance, folk customs, sightseeing activities, rural scene, greenway network, and cultural heritage. Their transformation ability was second only to actors with scores $>80$. Traditional skills and delicious food were on the edge of having a low transformation ability.

The centralization of a network is represented by the node distribution pattern. Low centralization indicates that the network nodes are scattered, with no aggregation. High centralization indicates that the central position of the network is occupied by a few nodes, presenting an uneven "center-edge." In this actor-network, the centralization of point out degree and point in degree were $22.6 \%$ and $21.1 \%$, respectively. This indicates that the actors had a low integration ability. Integration ability does not depend on the actor being in the core position of the network, but depends on the strength of the links between the actors. Accessibility level, folk customers, and the rural scene were the actors with strong transformation ability, and are generally important forces for a BRC.

2) The energy conversion efficiency in the network was unbalanced, giving the BRC actor-network different characteristics. The energy transformation efficiency $\mathrm{Q}$ value was mainly concentrated in three strong regions: a weak transition zone (38 - 49), medium conversion zone (50 - 59), and strong conversion zone (>60) (Figure 5). The actors with strong abilities included accessibility level, folk customs, the rural scene, sightseeing activities, community participation, 


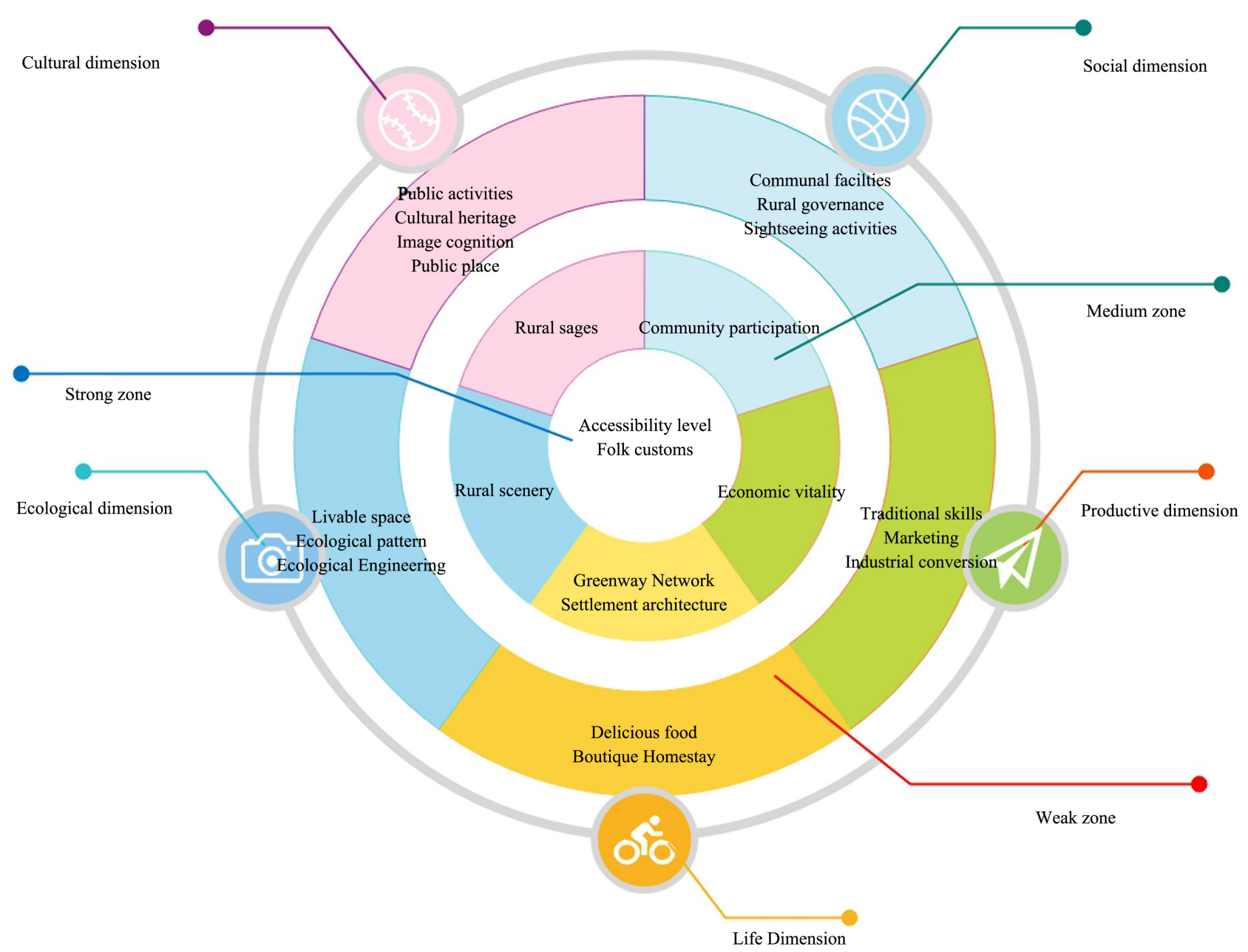

Figure 5. The circle structure of actors in beautiful rural construction network.

and the greenway network. These actors had a strong position and role in the network, and maintained exogenous contact. From an efficiency perspective, the abilities of actors in the network shape the structural characteristics. Actors in core positions have the advantages of transforming different internal and external elements into the endogenous network itself.

\subsection{Discussion}

1) From the perspective of ANT, EM reflects power relations, which includes government, collective organizations, villagers, tourists, capital, landscape, traditional culture, characteristic buildings, land, and all their interactions. EM is constrained by many actors, including both tangible commercial entities and different tangible and intangible actors affecting the BRC. Actors who promote rural diversification choose a diversified mode, form a diversified life pattern among rural residents, and promote the development of rural agriculture in the direction of multifunctional agriculture. Each actor may "accumulate" a certain amount of energy, and may be the source of external power for other actors. The energy of actors may come from their own historical accumulation or from their 
internal innovation transformation.

For example, with improvements in external and internal accessibility level, DLS gains significant energy from the highway exit to the high-speed railway station, facilitating the accessibility level of rural space for tourists and improving the shared experiential value of rural space. Constructing an ecological experience space network in DLS also creates an innovative pattern that improves the ecological livability of the space. This highlights the rural scenery, folk customs, and other characteristics. By keeping in close contact with other BRC elements, more kinetic energy is created, leading to more influential actors, and promoting high-quality BRC construction.

2) From a space production perspective, cultivating the actor network is inseparable from constructing rural space. Constructing a space in a creative form provides convenient conditions to transform actor roles. With the transformation and upgrades to rural tourism, non-human rural actors have new roles that extend the agricultural value chain in the network. The increased diversity in local livelihoods changes the structure of rural residential space and leisure space, advancing the diversification of rural space production. Introducing high technology and creating an innovation space can enable actors with strong transformation ability to establish more extensive contact with other actors on the Internet, transferring power between actors. A digital rural space helps create a diversified social intelligent technical support system, allowing more actors to accumulate a greater transformation ability, and to facilitate the transformation from traditional production-oriented agriculture to modern multifunctional agriculture. The EM transformation involves breaking through the narrow economic rationality that holds that "rural industry is agricultural production," and advancing the transformation of production-oriented agriculture into multifunctional agriculture. Implementing a rural innovation project builds a platform for the transformation of innovative knowledge. This emphasizes training local people on the use of the Internet, IoT, and intelligent agricultural technology. Modern technological knowledge services are provided to construct an intelligent rural environment, using new media technology.

3) A new EM can provide new development ideas for BRC, integrating the internal and external resources of industry, society, culture, and ecology based on the existing rural space. This can unify both endogenous and exogenous development. Relying on innovative mechanisms for land property rights, building innovative rural communities, maximizing the participation of different enthusiastic actors, and realizing the integration of regional culture can enhance the endogenous transformation of spatial actors. Guided by the construction of a shared experience value chain, this facilitates the integration of the modern agricultural productive process space and landscape space network, promoting transformation of agricultural value chain from a low-end to high-end direction. It is critical to combine external resource injections with the flow of urban and rural elements, and to construct an urban-rural integration development platform. This will help strengthen the protection of intellectual property rights and 
reduce the risk of losing different actors when developing sharing mechanisms. Planning and constructing an intelligent space can enrich the value of shared experiential activities, enhance the ability of different actors to innovate and transform, advance the sustainability of rural diversified livelihoods, and build a high-quality rural space.

\subsection{Conclusion}

1) EM is a complex and progressive network process which is continuously generated by different actors. The actors form the network shape the ability to change and activate innovation; the actors also obtain energy from the network and may create new values. The balanced mechanisms established by the network can provide each actor access to more internal and external forces, potentially strengthening their interaction with other participants in the network. As long as the positions of key actors in the network change, actor-network goals will also change, through the linkage effect between network nodes. If actors having a strong influence on other actors have a strong development orientation, they become key actors of EM transformation. Accessibility level, folk customs, rural scenery, and other actors constitute the "engine" of the transformation of the old and new modes, and become a power source that can activate other subjects to participate in rural development, however, they can only be the key node actors at a specific stage of EM. The actors with a higher level of efficiency generally exhibit strong network cohesion, and can bridge the cultural gap between actors and space production in different societies. Through innovations such as informatization, digitization, intelligence, and innovation, we should strengthen the leading role of the innovative actor and promote the transformation of the whole network. Moreover, it is important to build new business forms and development modes with shared experiences as core, and expand the transformation and communication effect of the network.

2) EM requires both modern agricultural and spatial integration to achieve high-quality construction, thus improving the governance capacity of rural areas. An innovative urban-rural mode of cooperation between rural communities and external organizations can improve the transformation ability of network actors, supporting the EM construction level. Meeting the common interests of network actors requires the integration of heterogeneous actors with common interest demands, cultivating actors with strong innovation abilities, and promoting connections between actors. Establishing green development as a core common interest may guide the coordination of both social and ecological rationality, ultimately leading to the network to a high-quality EM. Full attention should be given to the leading role of actors with strong abilities to strengthen the interaction between the main actors, promote the energy transformation from micro to macro, and improve the efficiency of BRC. By carrying forward the endogenous power led by rural sages, the local endogenous power can improve the level of rural endogenous governance. Actors in the network should 
take measures to promote the transformation of low-intensity actors, such as those creating traditional handicrafts, with special skills and/or special food knowledge, into powerful "driving converters." It is important to extend the agricultural value chain in an innovative way, integrating rural production with living and ecological actors, and enhancing the emotional value and shared value for all network participants.

3) It is important to provide diversified space to support overall network transformation by innovating the form of experience space, and providing diversified space opportunities for EM innovation. Constructing an innovative experience space involving stakeholders helps transform different resources into a driving force for rural tourism. It also enhances the transformative abilities of local industry, folk customs, architecture, landscape, culture, and other elements. We should build a characteristic rural governance mechanism, adhere to the normalization of rural community training and education, and improve community participation in BRC. Combining "top-down" guidance and cultivating conscious "bottom-up" knowledge allows rural communities to combine intellectual support with community participation; improves the efficiency of the knowledge sharing of modern agricultural public knowledge, quasi-public knowledge, and private knowledge to local farmers; improve farmer contributions to agriculture; and transform the ecological experience space.

\section{Acknowledgements}

This project was funded by the National Social Science Fund Project of China (15BGL118).

\section{Conflicts of Interest}

The authors declare no conflicts of interest regarding the publication of this paper.

\section{References}

Ai, S.-W., \& Miao, C.-H. (2010). "Space of Places", "Space of Flows" and "Space of Actor-Networks": From the Perspective of ANT. Human Geography, 25, 43-49.

Allen, C. D. (2011). On Actor-Network Theory and Landscape. Area, 43, 274-280. https://doi.org/10.1111/j.1475-4762.2011.01026.x

Barnes, M. L., Wang, P., Cinner, J. E., Graham, N. A. J., Guerrero, A. M., Jasny, L., Lau, J., Sutcliffe, S. R., \& Zamborain-Mason, J. (2020). Social Determinants of Adaptive and Transformative Responses to Climate Change. Nature Climate Change, 10, 823-828. https://doi.org/10.1038/s41558-020-0871-4

Chen, C., Woods, M., Chen, J. L., Liu, Y. Q., \& Gao, J. L. (2019). Globalization, State Intervention, Local Action and Rural Locality Reconstitution-A Case Study from Rural China. Habitat International, 93, Article ID: 102052.

https://doi.org/10.1016/j.habitatint.2019.102052

Chen, H. S., \& Wang, X. P. (2019). Exploring the Relationship between Rural Village Characteristics and Chinese Return Migrants' Participation in Farming: Path Dependence in Rural Employment, Cities, 88, 136-143. 
https://doi.org/10.1016/j.cities.2019.01.016

Chen, K. Q., Long, H. L., Liao, L. W., Tu, S. S., \& Li, T. T. (2020). Land Use Transitions and Urban-Rural Integrated Development: Theoretical Framework and China's Evidence. Land Use Policy, 92, Article ID: 104465.

https://doi.org/10.1016/j.landusepol.2020.104465

Cumming, G. S., \& Stephan, C. T. (2018). Linking Economic Growth Pathways and Environmental Sustainability by Understanding Development as Alternate Social-Ecological Regimes. Proceedings of the National Academy of Sciences of the United States of America, 115, 9533-9538. https://doi.org/10.1073/pnas.1807026115

Gao, C. L., \& Cheng, L. (2020). Tourism-Driven Rural Spatial Restructuring in the Metropolitan Fringe: An Empirical Observation. Land Use Policy, 95, Article ID: 104609. https://doi.org/10.1016/j.landusepol.2020.104609

Gao, J., \& Wu, B. H. (2017). Revitalizing Traditional Villages through Rural Tourism: A Case Study of Yuanjia Village, Shaanxi Province, China. Tourism Management, 63, 223-233. https://doi.org/10.1016/j.tourman.2017.04.003

Gautam, Y., \& Andersen, P. (2016). Rural Livelihood Diversification and Household Well-Being: Insights from Humla, Nepal. Journal of Rural Studies, 44, 239-249. https://doi.org/10.1016/j.jrurstud.2016.02.001

Ge, D. Z., Long, H. L., Qiao, W. F., Wang, Z. W., Sun, D. Q., \& Yang, R. (2020). Effects of Rural-Urban Migration on Agricultural Transformation: A Case of Yucheng City, China. Journal of Rural Studies, 76, 85-95. https://doi.org/10.1016/j.jrurstud.2020.04.010

Huang, Y. L., \& Yu, X. (2010). Identification of Key Elements of Regional Innovation Environment Based on Social Network Analysis. Journal of Beijing University of Posts and Telecommunications: Social Science Edition, 12, 84-89.

Jungsberg, L., Copus, A., Herslund, L. B., Nilsson, K., Perjo, L., Randall, L., \& Berlina, A. (2020). Key Actors in Community-Driven Social Innovation in Rural Areas in the Nordic Countries. Journal of Rural Studies, 79, 276-285.

https://doi.org/10.1016/j.jrurstud.2020.08.004

Kuang, F. Y., Jin, J. J., He, R., Wan, X. Y., \& Ning, J. (2019). Influence of Livelihood Capital on Adaptation Strategies: Evidence from Rural Households in Wushen Banner, China. Land Use Policy, 89, Article ID: 104228.

https://doi.org/10.1016/j.landusepol.2019.104228

Law, J. (1992). Notes on the Theory of the Actor-Network-Ordering. Strategy and Heterogeneity, Systems Practice, 5, 379-393. https://doi.org/10.1007/BF01059830

Li, L. H., Fu, D. F., \& Liu, R. (2014). Spatial Turn of Tourism Studies: A Review on Tourism Studies from the Perspective of the Actor-Network Theory. Tourism Tribune, 29, 107-115.

McKitterick, L., Quinn, B., McAdam, R., \& Dunn, A. (2016). Innovation Networks and the Institutional Actor-Producer Relationship in Rural Areas: The Context of Artisan Food Production. Journal of Rural Studies, 48, 41-52. https://doi.org/10.1016/j.jrurstud.2016.09.005

Richter, R. (2019). Rural Social Enterprises as Embedded Intermediaries: The Innovative Power of Connecting Rural Communities with Supra-Regional Networks. Journal of Rural Studies, 70, 179-187. https://doi.org/10.1016/j.jrurstud.2017.12.005

Rodger, K., Moore, S. A., \& Newsome, D. (2009). Wildlife Tourism, Science and Actor Network Theory. Annals of Tourism Research, 36, 645-666.

https://doi.org/10.1016/j.annals.2009.06.001

Steen, J. (2010). Actor-Network Theory and the Dilemma of the Resource Concept in 
Strategic Management. Scandinavian Journal of Management, 26, 324-331. https://doi.org/10.1016/j.scaman.2010.05.003

Tang, Z. C. (2019). Rural Revitalization and Scientific Management in the Pearl River Delta-Scientific Decision Based on Scientific Rationality and Public Understanding. Global Transitions, 1, 241-250. https://doi.org/10.1016/j.glt.2019.11.003

Tödtling, F. (2020). Regional Development, Endogenous. In A. Kobayashi (Ed.), International Encyclopedia of Human Geography (2nd ed., pp. 303-308). Oxford: Elsevier. https://doi.org/10.1016/B978-0-08-102295-5.10121-0

Vlaev, I. (2018). Local Choices: Rationality and the Contextuality of Decision-Making. Brain Sciences, 8, 8. https://doi.org/10.3390/brainsci8010008

Wang, L., \& Xiao, S. Z. (2020). Tourism Space Reconstruction of a World Heritage Site Based on Actor Network Theory: A Case Study of the Shibing Karst of the South China Karst World Heritage Site. International Journal of Geoheritage and Parks, 8, 140-151. https://doi.org/10.1016/j.ijgeop.2020.05.005

Yin, S., Yang, X. J., \& Chen, J. (2020). Adaptive Behavior of Farmers' Livelihoods in the Context of Human-Environment System Changes. Habitat International, 100, Article ID: 102185. https://doi.org/10.1016/j.habitatint.2020.102185

Zang, Y. Z., Liu, Y. S., Yang, Y. Y., Woods, M., \& Fois, F. (2020). Rural Decline or Restructuring? Implications for Sustainability Transitions in Rural China. Land Use Policy, 94, Article ID: 104531. https://doi.org/10.1016/j.landusepol.2020.104531

Zheng, L. J. (2010). Construction of Eco-Experiencescape: A Case Study of Da Lishu Village in Dandong. Journal of Eastern Liaoning University (Sciences), 12, 36.

Zheng, L. J. (2017). Study on the Development of Rural Ecological Experience Tourism-A Case Study of Dandong (pp. 57-75). Beijing: Economic Science Press.

Zheng, L. J. (2018). Analysis on the Transformation and Upgrading of Rural Tourism Based on Actor-Network Theory. Social Scientist, No. 10, 91-97.

Zheng, L. J., \& Liu, H. Q. (2018). Identification of Focal Actors in The Translation of the Rural Tourism Actor-Network: A Case in China. Environmental Engineering and Management Journal, 17, 2812-2821. https://doi.org/10.30638/eemj.2018.180

Zheng, L. J., Wang, H. Y., Luo, Y. Y., \& Zhou, X. L. (2019). Multifunctional Landscape Actor-Network Construction from the Perspective of Rural Tourism Experiences. Journal of Landscape Research, 11, 79-86. 